\title{
LA PERCEPCIÓN DE LOS RESIDENTES SOBRE LOS IMPACTOS DEL TURISMO COMO PREDICTOR DE LA PARTICIPACIÓN SOCIAL
}

\author{
Carlos Gauna Ruiz de León * \& Ana Bertha Gómez Delgado**
}

Resumen: La percepción de la población local sobre los impactos del turismo es un tema relevante en los estudios académicos a través de abordajes cualitativos y cuantitativos. Este estudio se realizó en la zona metropolitana de Puerto Vallarta, Jalisco y Bahía de Banderas, Nayarit, donde el turismo es la actividad preponderante. Con una encuesta levantada a la población, se desarrolló un "Modelo de Ecuaciones Estructurales" (SEM) el cual determinan que los impactos del turismo explican de forma adecuada la percepción de la población local, como esta fundamentado en la literatura académica, con la aportación en este trabajo, que la percepción es un predictor del interés por resolver sus problemas de la comunidad. Para el caso de estudio, aunque existen diferencias en la integración de los impactos como percepción, no hay diferencia estadística significativa en la percepción como predictor del interés por participar en las dos poblaciones analizadas.

Palabras claves: Turismo; Percepción; Población Local; Impactos del Turismo.

\section{RESIDENTS' PERCEPTION OF THE IMPACTS OF TOURISM ASA PREDICTOR OF SOCIAL PARTICIPATION}

\begin{abstract}
Residents' perception of the impacts of tourism is a relevant issue in academic studies through both qualitative and quantitative approaches. This study was conducted in the metropolitan area of Puerto Vallarta, Jalisco and Bahía de Banderas, Nayarit, where tourism is the predominant activity. With a survey raised to the population, it was developed a "Structural Equations Model" (SEM) which determine that the impacts of tourism explain in an adequate way the perception of the local population, as it is based on academic literature, with the contribution in this work, that the perception is a predictor of the interest to solve their problems of the community. For the case study, although there are differences in the integration of impacts as perception, there is no significant statistical difference in the perception as a predictor of the interest in participating in the two analyzed populations.
\end{abstract}

Keywords: Tourism; Perception; Local Residents; Tourism Impacts.

\section{AS PERCEPÇÕES DOS RESIDENTES SOBRE OS IMPACTOS DOTURISMO COMO PREDITOR DE PARTICIPAÇÃO SOCIAL}

Resumo: A percepção da população local sobre os impactos do turismo é um tema relevante nos estudos acadêmicos através de abordagens qualitativas e quantitativas. Este estudo foi realizado na área metropolitana de Puerto Vallarta, Jalisco e Bahía de Banderas, Nayarit, onde o turismo é a atividade predominante. Com um levantamento da população, foi desenvolvido um "Modelo de Equação Estrutural" (SEM), que determinou que os impactos do turismo explicam adequadamente a percepção da população local, pois se baseia na literatura acadêmica, com a contribuição deste trabalho, essa percepção é um preditor de interesse na solução de seus problemas da comunidade. Para o estudo de caso, embora haja diferenças na integração dos impactos como percepção, não há diferença estatística significativa na percepção como preditor de interesse em participar das duas populações analisadas.

Palavras-chave: Turismo; Percepção; População Local; Impactos do Turismo.

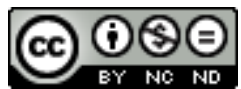

Licenciada por Creative Commons Atribuição Não Comercial / Sem Derivações/4.0/Internacional

\begin{abstract}
* Doctor en Cooperación y Bienestar Social por la Universidad de Málaga, España, Cum Laudem y Premio Extraordiandrio de Doctorado 2008, Licenciado en Economía por la Universidad de Guadalajara, Profesor Investigador de Tiempo completo Titular “ C' de la Universidad de Guadalajara, Miembro del Sistema Nacional de Investigadores Nivel I, Profesor con perfil deseable PRODEP, Coordinador del Doctorado en Ciencias para el Desarrollo, la Sustentabilidad y el Turismo, Profesor del Posgrado en Ciencias para el Desarrollo, la Sustentabilidad y el Turismo (Maestría y Doctorado) de la Universidad de Guadalajara y Miembro del Centro de Investigación CEDESTUR del Cucosta de la Universidad de Guadalajara. Orcid http://orcid.org/0000-0002-8325-9397 [carlosgaunaruiz@gmail.com ]

** Doctora en Ciudad, Territorio y Sustentabilidad por la Universidad de Guadalajara, Maestra en Desarrollo Turístico Sustentable por la Universidad de Guadalajaray Licenciada en Economía por la Universidad Autónoma de Nayarit, Profesor Investigador de Tiempo Completo Titular "B" de la Universidad de Guadalajara, Profesor con perfil deseable PRODEP, Profesor del Posgrado en Ciencias para el Desarrollo, la Sustentabilidad y el Turismo (Maestría y Doctorado) de la Universidad de Guadalajara y Miembro del Centro de Investigación CEDESTUR del Cucosta de la Universidad de Guadalajara. Orcid https://orcid.org/0000-0003-4943-8399 [ anabgomez1@ @hotmail.com]
\end{abstract}




\section{INTRODUCCIÓN}

El interés por conocer la percepción de la población local con respecto a los impactos del turismo es un tema que ha sido abordado desde diversas orientaciones académicas, el principio básico es que para que la actividad turística funcione de manera adecuada se requiere de la participación de los residentes y para que esto sucede es imperativo que los habitantes locales perciban que son mayores los beneficios que los costos sociales, si esto no sucede puede enfrentar al rechazo y con ello cancelar la posibilidad de éxito de los proyectos turísticos.

La toma de decisiones por parte de los agentes públicos o privados deben tomar en cuenta a los residentes locales, el éxito y la sostenibilidad de los proyectos depende de este apoyo (Andriotis y Vaughan, 2003), los habitantes locales de los destinos turísticos deben percibir que en el proceso de apoyo a la actividad turística reciben beneficios por su participación (Ap, 1992) y también evalúan en su percepción los costos que esto representa para la población (Gursoy y Rutherford, 2004).

Las variables que se usan para explicar la percepción de los habitantes locales tienen una marcada tendencia hacía los criterios económicos (ingreso y empleo), pero cada vez se usan criterios sociales, como el lugar de residencia, el origen étnico, la edad y la forma como se relacionan con los turistas (Harrill, 2004). Los impactos positivos y negativos a la cultura y la identidad de los habitantes locales es un elemento importante, de forma espacial en los sitios patrimonio de la humanidad (Meimand et al., 2017; Rasoolimanesh, Jaafar, Kock y Ahmad, 2017; Olya, Alipour y Gavilyan, 2018).

Las poblaciones locales no son entes comunes, con una sola percepción, dentro de cada grupo existen diversos grupos que tendrán percepciones diferentes, lo que hace complejo el análisis (Sánchez-Fernández, Álvarez-Bassi y Cardona, 2016).

El espacio donde viven los habitantes locales, natural o construido es usado para la realización de la actividad turística, allí se dará la interrelación entre turistas y residentes (Campodónico, 2016), en este proceso se generan impactos al espacio que el residente percibe como efectos de la actividad turística, no sólo negativos, degradación medioambiental, también positivos, construcción de infraestructura que mejora la calidad de vida de los locales (lqbal, 2020).
La actividad turística en la región conurbada de Puerto Vallarta-Bahía de Banderas es la principal actividad económica, recibe más de cuatro millones de visitantes al año, siendo el segundo destino de playa del país y el más importante del pacífico mexicano, genera el mayor número de empleos directos e indirectos, el mayor número de emprendimientos económicos y la ocupación espacial de forma directa se da en una amplía zona de la franja costera y el resto del territorio es usada para la habitación de los residentes, lo que provoca una amplia relación entre los habitantes locales y los turistas.

El desarrollo de la actividad turística ha generado un crecimiento poblacional por arriba de la media nacional, que implica una dinámica económica y social compleja, pues se tiene un constante requerimiento de atención a la población.

El turismo genera impactos positivos y negativos, que se pueden identificar en las dimensiones económicas, sociales, culturales y medioambientales, que deben ser estudiados para identificar a través de la percepción de los habitantes como se da su relación con la actividad turística, toda vez que los estudios sobre esta temática demuestran la importancia que tiene este proceso para el desarrollo del turismo y debe ser uno de los principales insumos para la toma de decisiones de los gestores públicos y privados.

El presente trabajo tiene como objetivo analizar si la percepción de los residentes sobre los impactos asociados al turismo (económicos, sociales, culturales y ambientales) es un predictor para que se genere interés por trabajar en la solución de los problemas. El trabajo empírico se realizó en los municipios de Bahía de Banderas, Nayarit (BB) y Puerto Vallarta, Jalisco (PVR), para los cual se estableció un modelo de ecuaciones estructurales (SEM) que permitió identificar las relaciones propuestas.

Al construir el modelo en las dos localidades se pretende identificar las diferencias que existen en los dos municipios, a partir de su estructura sociodemográfica. Los resultados que se presentan tienen el mismo sentido que los ofrecen los trabajos previos analizados, de forma particular los que trabajan mediante el modelado de ecuaciones estructurales (SEM), permiten identificar de manera adecuada, que la percepción de la población local a partir de los factores determinados por los tipos de impactos que genera el turismo son un predictor para identificar el nivel de interés por participar en la solución de los problemas que identifican los residentes. 
Con la aportación se puede explicar la percepción de las dos poblaciones estudiadas, identificar las diferencias y similitudes para entender la dinámica de cada una de ellas, lo que permitirá en estudios posteriores y en procesos de planeación del desarrollo tomar en cuenta las condiciones de la población que se generan en la región. Una aportación adicional es que se requieren estudios sobre esta temática en localidades turísticas de países en desarrollo, usando una metodología construida desde la experiencia académica de la realidad de la región.

\section{CUADRO TEÓRICO}

La percepción de los habitantes locales sobre los impactos del turismo es relevante porque son actores fundamentales en el proceso de la actividad en el territorio, pues allí viven y en algunos casos ha sido su lugar de residencia por generaciones, han generado una cultura, tradiciones y convivido con el medio natural a partir de las actividades productivas desarrolladas.

El estudio de la percepción de la población local sobre los impactos que genera el turismo ha sido estudiado desde diversos enfoques, es uno de los temas de mayor incidencia desde la academia, a partir de las aportaciones pioneras de Doxey (1975), Pizam (1978), Ap (1992), Andriotis y Vaughan (2003) Gursoy y Rutherford (2004), Harril (2004) y Nonkoo, et al. (2013) se creó una importante actividad académica para identificar las actitudes, motivaciones, conflictos y la manera como participa del turismo la población residente de los destinos.

Los impactos del turismo se identifican con factores económicos, sociales, culturales y medioambientales y de acuerdo a la percepción que tienen sobre estos los habitantes locales actuan desde su posición en la comunidad a favor del desarrollo turístico sostenible (Ap, 1992; Vargas-Sánchez, PorrasBueno y Plaza-Mejía, 2011; Nunkoo y Gursoy, 2012; Ribeiro, Pinto, Silva y Woosnam, 2017). Una condición para que esto suceda es que los beneficios excedan a los costos (Andereck y Vogt, 2000; Andereck, Valentine, Knopf y Vogt, 2005; Nunkoo y Gursoy, 2012).

La teoría del intercambio social es adecuada para identificar como se desarrolla un proceso de intercambio a partir del turismo, donde las comunidades ofrecen apoyar y trabajar para los visitantes con el interés de recibir beneficios que les mejoren su condición de vida (Ap, 1992), si los habitantes perciben que los beneficios son mayores que los costos, se sienten inclinados a participar en el intercambio y aprueban el desarrollo futuro de su comunidad (Ko y Stewart, 2002; Gursoy y Rutherford, 2004;), la percepción que tienen los habitantes y las actitudes hacía los beneficios y los costos asociados al turismo influyen en el apoyo al desarrollo turístico sostenible (Vargas-Sánchez et al., 2011; Nunkoo y Gursoy, 2012).

Andereck et. al. (2005) realizan un análisis de la percepción de los residentes, teniendo diferentes opiniones entre los que tienen una actividad relacionada con el turismo y los que su actividad no es prioritaria para el turismo.

Una critica que se tiene sobre el uso de la teoría del intercambio social ha sido que esta propuesta se basa en la idea de que los grupos sociales que intervienen lo hacen de forma homogénea, de forma particular con grupos diversos que funcionan en las comunidades locales y tienen diversas motivaciones (Sánchez-Fernández et al., 2016), se han implementado estudios que plantean el uso de otras teorías de forma conjunta, desde la teoría de la identidad, que propone que se debe analizar a la población local a partir de segmentarla por grupos dependiendo alguna tipología específica, como puede ser su orientación religiosa (Meimand et al., 2017), su apego comunitario (Rasoolimanesh, Jaafar, etal., 2017; Eusébio, Vieira y Lima, 2018; Olya et al., 2018;) y el involucramiento en la actividad turística ( Ribeiro et al., 2017; Ouyang, Gursoy y Chen, 2019).

Nunkoo, Smith y Ramkosoon (2013) en una primera aproximación al estado de la cuestión, realizan un análisis de los trabajos publicados de 1984 al 2010 en tres revistas académicas de importancia "Annals of Tourism Research", "Journal of Travel Research" y "Tourism Management", hacen una distribución entre los trabajos que usan alguna orientación teórica y los métodos cuantitativos o cualitativos que aplican en su trabajo, más $50 \%$ de los trabajos revisados tienen como sustento teórico a la teoría del intercambio social y el $72 \%$ de los trabajos usan métodos cuantitativos.

Sharpley (2014) sintetiza lo que hasta ese momento se había generado en la temática de la percepción de la población, haciendo una crítica en tres aspectos: al planteamiento teórico que se presenta porque no se realizan análisis transdisciplinares, no existen aportaciones teóricas importante en cuanto al estudio del turismo, la mayor parte de los trabajos no 
abonan a la planeación de la actividad turística, por la limitante en la aplicación en áreas que difícilmente pueden ser usadas como referentes en otros destinos (gran parte se realizan en países desarrollados) y porque la mayoría de las metodologías utilizadas son cuantitativas, al usar una serie de variables controladas, que solo explican lo que perciben los residentes, pero no analizan porque tienen esas percepciones.

\section{METODOLOGÍA}

En la preparación del presente estudio, se hizo la revisión del estado de la cuestión, usando cinco palabras claves "host", "resident", "perception",

Tabla 1. País de origen de los artículos publicados

\begin{tabular}{|l|l|l|c|}
\hline País & Total & País & Total \\
\hline Estados Unidos & 39 & Malasia & 7 \\
\hline China & 17 & Sudáfrica & 6 \\
\hline Australia & 14 & Corea del Sur & 6 \\
\hline Inglaterra & 13 & Canadá & 4 \\
\hline España & 11 & Mauricio & 2 \\
\hline
\end{tabular}

Fuente: elaboración propia.

Los principales autores son Gursoy (8 artículos), Nunkoo (5 artículos), Rassolimanesh (5 artículos), Woosman (5 artículos), Jaffar (4 artículos), Monterrubio ( 3 artículos) y Ramkinson (3 artículos), la mayor parte de ellos además son citados de forma amplía en otros trabajos, existen diversos abordajes, tanto cualitativos como cuantitativos.

En estos trabajos se sintetizan las preocupaciones que tienen las poblaciones locales en los lugares que se realiza turismo, su participación en la actividad turística y la forma como se modifican las formas sus vida por la llegada de los turistas, por la creación de nuevas empresas y los cambios en la conformación demográfica de las poblaciones de acogida, un tema recurrente es la utilización del territorio, que es convertido en atractivo del turismo, que es transformado para su uso intensivo provocando impactos negativos, contrarios al principio del "Desarrollo Sostenible".

Tabla 3. Revista en que fueron publicados artículos con SEM

\begin{tabular}{|l|l|}
\hline Tourism Managment & 3 \\
\hline Annals of Tourism Research & 3 \\
\hline Journal of Travel Research & 3 \\
\hline Sustainability & 4 \\
\hline Journal of Sustainability Tourism & 4 \\
\hline
\end{tabular}

Fuente: elaboración propia. "attitudes" y "tourism" en la base de datos "Web of science", haciendo énfasis en las cinco principales revistas que más estudios tienen bajo estos criterios, "Annals of Tourism Research", "Journal of Travel Research" y "Tourism Management", "Sustainaibity" y "Journal of Sustainability Tourism", que fueran artículos científicos publicados los últimos 10 años (2010-2020), este trabajo es similar al realizado por Nunkoo et. al. (2013).

Se tuvo como resultado 106 artículos científicos, de los cuales 60 (58.2\%) han sido publicados los últimos 6 años (a partir de 2015). Esto demuestra la importancia que ha tenido el tema en el campo de la investigación.

Tabla 2. Revista en que fueron publicados

\begin{tabular}{|l|l|}
\hline Tourism Managment & 32 \\
\hline Annals of Tourism Research & 22 \\
\hline Journal of travel Research & 19 \\
\hline Sustainability & 16 \\
\hline Journal of Sustainability Tourism & 14 \\
\hline
\end{tabular}

Fuente: elaboración propia.

De esta revisión se seleccionaron aquellos trabajos que utilizaron métodos cuantitativos, de forma particular a través del "Modelo de Ecuaciones Estructurales" (SEM), en esta categoría se identificaron 17 artículos.

Los trabajos que se han realizado sobre la percepción de los habitantes locales y que usaron como proceso del trabajo empírico Modelos de Ecuciones Estructurales (SEM) tienen como principal coincidencia que permiten demostrar la importancia que tienen los impactos económicos sociales, culturales y medioambientales, ya sean beneficios o costos, sobre los intereses de las poblaciones.

La mayoria de ellas toman a la teoria del Intercambio social como base teórica (Vargas-Sánchez et al. 2011; Nunkoo \& Gursoy, 2012; Meimand et al., 2017; Rasoolimanesh, Jaafar, etal., 2017; Rasoolimanesh, Ringle, et al., 2017; Rasoolimanesh, Roldán, et al., 2017; Ribeiro et al., 2017; Wang \& Chen, 2015; Yu et al., 2018; Olya et al., 2018; Eusébio et al., 2018; Rasoolimanesh et al., 2019; Juric et al., 2020).

Debido a que existen criticas a la teoría del intercambio social porque los grupos que se relacionan no pueden tener criterios únicos y homogéneos, algunos trabajos han usado de forma agregada alguna otra teoría de forma particular la Teoría de la Identidad, que permite explicar como desde diversos grupos, aun 
que existen diferencia, que pueden ser demostradas, éstas afinan de mejor manera la percepción de las comunidades (Nunkoo y Gursoy, 2012; Rasoolimanesh, Jaafar, et al., 2017; Rasoolimanesh, Ringle, et al., 2017; Meimand et al., 2017; Eusébio et al., 2018; Olya et al., 2018; Rasoolimanesh et al., 2019).

Algunos trabajos usan de forma adicional otras teorías para formular sus trabajos como la Teoría de la Autodeterminación (Meimand et al., 2017), la Teoría de la Calidad de Vida (Ouyang et al., 2019), dos usan la Teoría de la Solidaridad Social (Woosnam, 2012;Juric et al., 2020), la Teoría de la Acción Razonada (Liao, Lin y Hsieh, 2019) y la Teoría del Ciclo de Vida de los Destinos (Vargas-Sánchez et al., 2015).
Las principales aportaciones de los trabajos seleccionados se presentan en la tabla 4, lo que permite demostrar que los Modelos de Ecuaciones Estructurales tienen la capacidad de dar información valiosa de la percepción de la población local sobre la actividad del turismo, según el enfoque de cada investigación se pueden explicar las condiciones bajo las cuales los habitantes toman decisiones que influyen en la actividad turística, sus motivaciones que en la mayor parte son económicas pero también han visto que son importantes para la protección de su patrimonio natural o cultural.

\section{Tabla 4 Principales aportaciones de los trabajos académicos que usan Modelos de Ecuaciones Estructurales (SEM)}

\begin{tabular}{|c|c|c|}
\hline \multirow[b]{2}{*}{ Temática } & \multirow{2}{*}{\multicolumn{2}{|c|}{ Aportaciones }} \\
\hline & & Autores \\
\hline \multirow{3}{*}{$\begin{array}{l}\text { Factores que } \\
\text { determinan el } \\
\text { apoyo al } \\
\text { desarrollo turístico }\end{array}$} & $\begin{array}{l}\text { La construcción de un modelo que permita explicar el proceso } \\
\text { mediante el cual los habitantes locales participan en el turismo, } \\
\text { según el nivel de desarrollo del turismo en la localidad. }\end{array}$ & $\begin{array}{l}\text { Vargas-Sánchez et al. (2011); } \\
\text { Vargas-Sánchez et al. (2015) }\end{array}$ \\
\hline & $\begin{array}{l}\text { Factores económicos y no económicos que perciben los } \\
\text { habitantes locales y que influyen en su participación al desarrollo } \\
\text { del turismo. }\end{array}$ & $\begin{array}{c}\text { Nunkoo y Gursoy (2012); Woosnam, } \\
\text { (2012); Wang y Chen (2015); Ribeiro } \\
\text { et al. (2017); Meimand et al. (2017); } \\
\text { Rasoolimanesh, Jaafar, et al. (2017); } \\
\text { Rasoolimanesh, Ringle, et al. (2017); } \\
\text { Yu et al. (2018); } \\
\text { Eusébio et al. (2018); Olya et al. } \\
\text { (2018); Liao et al. (2019); Kuščery } \\
\text { Mihalič, (2019); Rasoolimanesh et } \\
\text { al.,(2019); Ouyang et al., 2019) }\end{array}$ \\
\hline & La interacción anfitrión- turista. & $\begin{array}{l}\text { Eusébio et al. (2018); Kuščery } \\
\text { Mihalič (2019) }\end{array}$ \\
\hline $\begin{array}{l}\text { Participación } \\
\text { directa en el } \\
\text { turismo }\end{array}$ & $\begin{array}{c}\text { Las personas participan en el turismo tienen un interés personal } \\
\text { en la actividad y perciben un beneficio, lo que permite un mayor } \\
\text { nivel de apoyo para el desarrollo del turismo dentro de su } \\
\text { comunidad. }\end{array}$ & $\begin{array}{c}\text { Woosnam (2012); Vargas- } \\
\text { Sánchez et al. (2011); } \\
\text { Vargas-Sánchez et al. (2015); Ribeiro } \\
\text { et al. (2017) }\end{array}$ \\
\hline $\begin{array}{c}\text { Criterios } \\
\text { medioambientales }\end{array}$ & $\begin{array}{c}\text { En su análisis toman como factores el uso del medio ambiente } \\
\text { natural y su relación de la comunidad. }\end{array}$ & $\begin{array}{c}\text { Nunkoo y Gursoy (2012); (Woosnam } \\
\text { (2012); Rasoolimanesh, Jaafar, et al., } \\
\text { (2017); Rasoolimanesh, Ringle, et al. } \\
\text { (2017); Rasoolimanesh et al. (2019) } \\
\text { Liao et al. (2019); Kuščer y Mihalič } \\
\text { (2019) }\end{array}$ \\
\hline \multirow{2}{*}{$\begin{array}{c}\text { Criterios } \\
\text { socioculturales }\end{array}$} & $\begin{array}{l}\text { Los habitantes locales que profesan la religión tienen mayor } \\
\text { compasión por el turismo, pero en general sacrifican sus } \\
\text { creencias por los beneficios económicos. }\end{array}$ & Meimand et al. (2017) \\
\hline & Usan factores a partir de la problemática social. & $\begin{array}{l}\text { Meimand et al. (2017); Liao et al. } \\
\text { (2019); Kuščer y Mihalič (2019) }\end{array}$ \\
\hline \multirow{2}{*}{$\begin{array}{l}\text { Sitios patrimonio } \\
\text { mundial de la } \\
\text { Humanidad }\end{array}$} & $\begin{array}{l}\text { Los habitantes muestran un apego a su lugar y a partir de los } \\
\text { impactos percibidos, están dispuestos a generar apoyos para la } \\
\text { conservación del patrimonio y el desarrollo turístico. }\end{array}$ & $\begin{array}{l}\text { Rasoolimanesh, Jaafar, et al.(2017); } \\
\text { Rasoolimanesh, Ringle, et al. (2017); } \\
\quad \text { Rasoolimanesh et al. (2019). }\end{array}$ \\
\hline & $\begin{array}{l}\text { El desarrollo de las actividades turísticas en los sitios patrimonio } \\
\text { generan beneficios económicos y fortalecen el apego a la } \\
\text { comunidad que procura el cuidado de los espacios culturales. }\end{array}$ & Olya et al. (2018) \\
\hline
\end{tabular}




\begin{tabular}{|c|c|c|}
\hline & $\begin{array}{l}\text { En zonas donde las comunidades son indígenas, el desarrollo } \\
\text { turístico refuerza la autoestima y provoca una cultura de } \\
\text { preservación de su cultura y tradiciones. }\end{array}$ & Liao et al. (2019) \\
\hline \multirow{3}{*}{$\begin{array}{l}\text { Turismo asociado } \\
\text { a eventos } \\
\text { deportivos }\end{array}$} & $\begin{array}{c}\text { Los residentes que participan en los eventos deportivos tienen } \\
\text { mejor actitud hacia los eventos, tienen una identidad ambiental y } \\
\text { de género positivas, a partir de que perciben que son más los } \\
\text { beneficios que los costos. }\end{array}$ & Nunkoo y Gursoy (2012) \\
\hline & $\begin{array}{l}\text { Los residentes que participan en los eventos de forma activa, } \\
\text { reciben más beneficios y están dispuestos a trabajar a favor del } \\
\text { desarrollo del turismo y ponderan los beneficios y minimizan los } \\
\text { impactos negativos. }\end{array}$ & $\begin{array}{l}\text { Wang y Chen, (2015); Ouyang et al. } \\
\text { (2019) }\end{array}$ \\
\hline & $\begin{array}{c}\text { La percepción de mejora en la calidad de vida y las afectaciones } \\
\text { en su entorno por el evento deportivo son elementos a tomar en } \\
\text { cuenta para evaluar el apoyo de los residentes al turismo } \\
\text { deportivo. }\end{array}$ & Yu et al. (2018) \\
\hline Overturismo & $\begin{array}{c}\text { Existen factores sobre la calidad de vida que son determinantes } \\
\text { en el rechazo al turismo (la contaminación del aire, el tráfico y el } \\
\text { hacinamiento) que influyen en la irritación de los residentes y } \\
\text { hace que los habitantes locales no apoyen los procesos de } \\
\text { desarrollo turístico, Como elementos positivos están la mejora de } \\
\text { la infraestructura relacionada con el turismo y la propia } \\
\text { comunidad y los beneficios económicos en los negocios de } \\
\text { hostelería en el destino. }\end{array}$ & Kuščer y Mihalič (2019) \\
\hline $\begin{array}{l}\text { Turismo no } \\
\text { monetarizado }\end{array}$ & $\begin{array}{l}\text { La solidaridad emocional hace posible este tipo de turismo, como } \\
\text { el carácter acogedor y la cercanía emocional, no así la simpatía } \\
\text { emocional. }\end{array}$ & Juric et al. (2020) \\
\hline $\begin{array}{l}\text { Criterios } \\
\text { psicológicos }\end{array}$ & $\begin{array}{l}\text { Utilizan en sus trabajos factores que provienen de la psicología, } \\
\text { para explicar las motivaciones de su participación. }\end{array}$ & $\begin{array}{c}\text { Woosnam (2012); } \\
\text { Wang y Chen, (2015); Juric et a l. } \\
\text { (2020) }\end{array}$ \\
\hline
\end{tabular}

Fuente: Elaboración propia a partir de la revisión bibliométrica.

Las investigaciones que se presentan usan una ubicación geográfica (Tabla 5), así encontramos trabajos en cuatro continentes, que permiten identificar en cada uno de ellos las condiciones de particulares de los habitantes, una critica generada por Sharpley (2014) era que la mayoría de las investigaciones se habían realizado en países desarrollados, pero en esta nueva revisión se observa que cada vez existen más trabajos en países en desarrollo, aunque ninguno de los que se presentan se realiza en Latinoamérica, lo que da una oportunidad de realizar estudios sobre percepción de los habitantes y su influencia en una actividad que ha sido ampliamente desarrollada en la región.

Tabla 5 Localización geográfica de los trabajos académicos que usan Modelos de Ecuaciones Estructurales (SEM)

\begin{tabular}{|c|c|c|}
\hline & Localidad/País & Autor \\
\hline \multirow[t]{2}{*}{ África } & Cabo Verde & Ribeiro et al. (2017); Eusébio et al. (2018) \\
\hline & Isla Mauricio & Nunkoo y Gursoy (2012) \\
\hline \multirow[t]{4}{*}{ Asía } & Taiwán & Liao et al. (2019); Meimand et al. (2017); \\
\hline & Malasia & $\begin{array}{l}\text { Rasoolimanesh, Jaafar, et al. (2017); Rasoolimanesh, Ringle, et al. (2017); } \\
\text { Rasoolimanesh et al. (2019) }\end{array}$ \\
\hline & Hong Kong & Ouyang et al. (2019) \\
\hline & Irán & Olya et al. (2018) \\
\hline \multirow[t]{4}{*}{ Europa } & Alemania & Juric et al. (2020) \\
\hline & Eslovenia & Kuščer y Mihalič (2019) \\
\hline & España & Vargas-Sánchez et al. (2011) \\
\hline & España/Portugal & Vargas-Sánchez et al. (2015) \\
\hline América & Estados Unidos da América & Woosnam, 2012); Wang y Chen (2015); (Yu et al. (2018) \\
\hline
\end{tabular}

Fuente: Elaboración propia a partir de la revisión bibliométrica. 
La revisión de literatura da cuenta de la importancia que tienen los trabajos que analizan la percepción de las poblaciones sobre los impactos del turismo, usando el modelo SEM, la formas como las comunidades identifican los beneficios y los costos que se producen en las dimensiones (factores) que se tienen identificados desde la base teórica. Las preocupaciones difieren de acuerdo a la tipología del turismo, el nivel de desarrollo de la actividad y de la estructura social y económica de la propia comunidad, por lo que no se pueden tomar como condiciones generales a situaciones que suceden en un tiempo y lugar determinado, pero que las aportaciones teóricas y metodológicas son adecuadas para el análisis de la problemática estudiada.

Las propuestas basadas en metodologías cuantitativas, tiene la limitante de la construcción de la muestra representativa, pero tienen a favor la demostración empírica que se fundamenta en pruebas estadísticas que le dan un nivel de certidumbre.

Los trabajos realizados en diversas comunidades dan una mayor fortaleza al planteamiento teórico, además que se puede conocer las condiciones particulares de los destinos estudiados, por ello la importancia de que este trabajo se realice en una región turística de México.

El presente estudio ofrece la posibilidad de comprender las condiciones similares y diversas que se dan en la región metropolitana de PVR-BB, su objetivo es evaluar sí la percepción de la población de los impactos del turismo (sociales, económicos, culturales y medioambientales) es un predictor del interés de los habitantes locales para resolver los problemas, analizando las condiciones de las dos poblaciones para identificar si existen diferencias en esta percepción.
En el mes de febrero de 2020 se aplicó el mismo instrumento en los dos municipios de manera independiente, a partir de los datos de la población (INEGI, 2010) la muestra resultó de 400 encuestas para cada municipio, se determinó un método polietápico de distribución, tomando en consideración los diferentes tipos de localidades de cada municipio, la distribución por sexo y grupos de edad, con un nivel de confianza del $95 \%$ y un grado de error de $+/-5 \%$. Se aplicó a personas mayores de edad y en los domicilios particulares de los entrevistados.

La encuesta incluye información sociodemográfica de la población, nivel de estudio, nivel de escolaridad, actividad económica que realizan, tipo de empresa donde laboran y tiempo que tienen de radicar en la región, además de preguntas sobre la percepción que tienen sobre los impactos del turismo en la población y el interés que tienen por participar en la solución de los problemas de la población, estas preguntas que son base del estudio son construidas en términos de la consideración del impacto, con escala Likert, de uno como mínimo y cinco como máximo.

Este instrumento se realizó a partir de la experiencia como investigadores y permite tomar en consideración algunos de los asuntos más críticos de la problemática asociada al turismo, se encuentran similitudes con los criterios utilizados en trabajos que se presentan en la revisión de la literatura.

Una vez que se capturó la información, se realizó el análisis en el programa estadístico SPSS (versión 23), los indicadores básicos (Tabla 6) permiten explicar que la muestra tiene datos proporcionales similares con los datos sociodemográficos presentados y de los datos de las variables con valores de estadísticas descriptivas (Tabla 7).

Tabla 6. Indicadores básicos de la toma de información de PVR-BB

\begin{tabular}{|c|c|c|c|c|c|}
\hline & \multicolumn{2}{|c|}{ Puerto Vallarta } & \multicolumn{2}{|c|}{ Bahía de Banderas } \\
\hline & & Frecuencia & $\%$ & Frecuencia & $\%$ \\
\hline \multirow{2}{*}{ Sexo } & Masculino & 200 & 50 & 200 & 50 \\
\hline & Femenino & 200 & 50 & 200 & 50 \\
\hline \multirow{3}{*}{ Edad } & 18 a 30 años & 120 & 30 & 120 & 30 \\
\hline & 31 a 65 años & 240 & 60 & 240 & 60 \\
\hline & Más de 65 años & 40 & 10 & 40 & 10 \\
\hline \multirow{6}{*}{ Escolaridad } & Sin estudios & 18 & 4.5 & 33 & 8.3 \\
\hline & Primaria & 60 & 15 & 61 & 15.3 \\
\hline & Secundaria & 103 & 25.8 & 110 & 27.5 \\
\hline & Preparatoria & 128 & 32 & 133 & 33.3 \\
\hline & Profesional & 83 & 20.8 & 59 & 14.8 \\
\hline & Posgrado & 8 & 2 & 4 & 1 \\
\hline Actividad & & & & & \\
\hline
\end{tabular}




\begin{tabular}{|c|c|c|c|c|c|}
\hline & Empresario & 38 & 9.5 & 55 & 13.8 \\
\hline & Empleado público & 74 & 18.5 & 69 & 17.3 \\
\hline & Empelado privado & 95 & 23.8 & 116 & 29 \\
\hline & Campesino & 4 & 1 & 14 & 3.5 \\
\hline & Trabajadorind. & 49 & 12.3 & 45 & 11.3 \\
\hline & Profesionista & 24 & 6 & 15 & 3.8 \\
\hline & Estudiante & 40 & 10 & 26 & 6.5 \\
\hline & Ama de casa & 46 & 11.5 & 40 & 10 \\
\hline & Desempleado & 16 & 4 & 11 & 2.8 \\
\hline & Jubilado & 14 & 3.5 & 9 & 2.3 \\
\hline \multirow{5}{*}{ Residencia } & Menos de 5 años & 59 & 14.8 & 44 & 11 \\
\hline & 6 y 10 años & 59 & 14.8 & 72 & 18 \\
\hline & Entre 11 y 15 años & 81 & 20.3 & 102 & 25.5 \\
\hline & Entre 16 y 20 años & 89 & 22.3 & 83 & 20.8 \\
\hline & Más de 21 años & 112 & 28 & 99 & 24.8 \\
\hline
\end{tabular}

Fuente: Elaboración propia a partir de los resultados del instrumento.

De acuerdo a la información que se observa en la tabla 7, las variables económicas sobre el empleo y el empleo remunerado tiene una correspondencia con lo que presentan Ap (1992), Gursoy y Rutherford (2004), Vargas-Sánchez et al. (2011), Rasoolimanesh, Jaafar, et al. (2017) y Ribeiro et al. (2017).

Sobre los impactos sociales, de forma particular sobre prostitución, alcoholismo y drogadicción se basa en lo presentado por Vargas-Sánchez etal. (2011), Nunkoo y Gursoy (2012), Rasoolimanesh, Jaafar, et al. (2017) y Ribeiro et al. (2017).

En cuanto a factores culturales sobre tradiciones, identidad y cultura local, se tiene coincidencia con Vargas-Sánchez et al. (2011), Nunkoo y Gursoy (2012), Rasoolimanesh, Jaafar, etal. (2017) y Ribeiro et al.
(2017), (Meimand et al. (2017), Olya et al. (2018) y Liao et al. (2019)

En cuanto a los factores que tienen sobre el medioambientales y los efectos que genera las actividades sociales, de forma particular del turismo, tienen como base en los estudios de Vargas-Sánchez et al. (2011) Nunkoo y Gursoy (2012), Rasoolimanesh, Jaafar, et al. (2017) y Ribeiro et al. (2017), Woosnam (2012) e lqbal (2020).

Sobre los intereses por participar en los procesos de desarrollo y apoyar en resolver los problemas que se generan en su comunidad, son similares a los criterios utilizados por Vargas-Sánchez et al. (2011), Nunkoo y Gursoy (2012), Rasoolimanesh, Jaafar, et al. (2017) y Ribeiro et al. y Eusebio (2018).

Tabla 7. Indicadores del modelo de acuerdo a los factores

\begin{tabular}{|c|c|c|c|c|c|}
\hline \multirow{2}{*}{ Variable } & \multirow{2}{*}{ Indicador } & \multicolumn{2}{|c|}{ Puerto Vallarta } & \multicolumn{2}{c|}{ Bahía de Banderas } \\
\cline { 3 - 6 } & Media & D. E. & Media & D. E. \\
\hline Interés por los problemas de la localidad & Interés & 3.59 & 1.259 & 3.77 & 1.171 \\
\hline $\begin{array}{c}\text { Participación resolver los problemas de la } \\
\text { comunidad }\end{array}$ & Participación & 3.53 & 1.419 & 3.59 & 1.340 \\
\hline $\begin{array}{c}\text { Creación de empleos } \\
\text { Creación de empleos remunerados y } \\
\text { permanentes }\end{array}$ & Empleos & 4.20 & 1.063 & 4.20 & 1.084 \\
\hline Incremento del Alcoholismo & Remunerados & 3.67 & 1.257 & 3.35 & 1.200 \\
\hline Incremento de la Drogadicción & Drogadicción & 3.69 & 1.428 & 3.38 & 1.395 \\
\hline Incremento de la Prostitución & Prostitución & 3.38 & 1.512 & 2.76 & 1.394 \\
\hline Pérdida de Identidad cultural & Identidad & 2.97 & 1.488 & 2.60 & 1.411 \\
\hline Pérdida de las tradiciones popularesy la cultura & Tradición & 3.24 & 1.369 & 3.11 & 1.278 \\
\hline $\begin{array}{c}\text { Pérdida de lugares de playa por erosión y por } \\
\text { construcciones en las áreas naturales }\end{array}$ & Erosión & 3.07 & 1.416 & 2.90 & 1.267 \\
\hline Destrucción de bosques yáreas verdes & Bosques & 4.62 & 0.820 & 4.34 & 1.076 \\
\hline Daños a los ecosistemas marinos & Ecosistemas & 4.55 & 0.866 & 4.10 & 1.236 \\
\hline
\end{tabular}

Fuente: Elaboración propia a partir de los resultados del instrumento. 
Se realizó un análisis factorial para ambas muestras, para conocer el comportamiento de las variables independientes y saber cómo se agrupan en factores latentes (Hair, Anderson, Tathan y Black, 2008; Luque, 2000; Malhortra, 2000). Se presentan la pruebas estadísticas del índice de Kaiser Mayer Olkin y la prueba de esfericidad de Bartlett para determinar que es adecuado el análisis factorial, agregado los valores de las comunalidades, que es la varianza que una variable comparte con las demás y cuyos valores deben ser superiores a 0.6 para ser válidos y se integran para cada factor de la prueba a la totalidad, los valores del estadístico de fiabilidad de acuerdo a la prueba "Alpha de Cronbach", que es un estadístico de fiabilidad de las escalas usadas, debiendo tener valores por arriba de 0.6.

Se trabajó en el programa AMOS SPSS versión 23, donde se construyen modelos de ecuaciones estructurales (SEM), para poder determinar cómo se identifican cada una de las variables e identificar el comportamiento de las variables observadas al integrarse en las variables latentes, que se supone deberán ajustar en la misma forma que en el modelo factorial, con el agregado que ahora se podrá determinar las cargas de regresión estandarizadas que permiten determinar el impacto que tiene cada variable independiente sobre la variable dependiente dentro del modelo completo, además como los factores exógenos actuan sobre los factores endógenos (Arburkle, 2015; Hair, et al. 2008; Luque, 2000), Para que esto sea posible es necesario que los modelos que se desarrollan tengan la idoneidad en su construcción a través de las pruebas estadísticas propuestas.

La condición de que este modelo permita generar explicaciones, se basa en que se han tomado solo algunas de las variables del problema que se plantea, de acuerdo a criterios teóricos fundados, no se puede generalizar como los únicos, porque de acuerdo a diversos abordajes pueden existir otros factores, al ser determinado a partir de una muestra representativa construida con criterios de validación estadística, el trabajo se basa en una serie de supuestos que permiten una mejor compresión del problema abordado, que deberá será nutrido por otros abordajes de diversa estructura y método.

\section{PRESENTACIÓN DEL CASO DE ESTUDIO}

La zona metropolitana de "Puerto Vallarta, Jalisco (PVR) - Bahía de Banderas, Nayarit" (BB) es parte de un destino turístico que recibe más de seis millones de turistas al año (Tabla 8), es el segundo destino de playa de México (después de Cancún, Quintana Roo).

Tabla 8. Turistas hospedados según localidad 2018

\begin{tabular}{|l|l|l|l|}
\hline & Nacionales & Extranjeros & Total \\
\hline Nuevo Vallarta, Nayarit & 796,912 & $2,006,526$ & $2,803,438$ \\
\hline Puerto Vallarta, Jalisco & $1,368,354$ & $1,963,646$ & $3,331,990$ \\
\hline Total & $2,165,266$ & $3,970,162$ & $6,135,428$ \\
\hline
\end{tabular}

Fuente: Datatur/SECTUR 2018.

La región tiene una gran cantidad de recursos turísticos, está integrada por una gran biodiversidad marítima y terrestre de una belleza singular, con un clima ideal para las actividades turísticas y tiene una gran tradición cultural, es una localidad con una imagen típica de una población de estilo mexicano.

La región tiene un crecimiento poblacional sostenido superior a la media nacional (Virgen y
Gauna, 2011), que la ha convertido en un polo de desarrollo, ambas localidades son de las más importantes en sus respectivos estados. En el año 2010 había cerca de trescientos ochenta mil personas viviendo (INEGI, 2010), sí se ha mantenido la tasa de crecimiento, en el año 2020 (una vez que se conozcan los datos del XIV censo de población y vivienda, que realiza en INEGI) se puede llegar a más de seiscientos mil habitantes (Tabla 9).

Tabla 9. Total de la Población y tasa de crecimiento de Puerto Vallarta-Bahía de Banderas 1990-2020.

\begin{tabular}{|l|c|c|c|c|}
\hline & Población & Tasa de crecimiento & Población & Tasa de crecimiento \\
\hline & 1990 & 2000 & 2010 & $2000-2010$ \\
\hline Bahía de Banderas & 39,831 & 59,808 & 124,209 & 10.77 \\
\hline Puerto Vallarta & 111,457 & 184,728 & 255,725 & 3.84 \\
\hline Total & 151,288 & 244,536 & 379,934 & 5.54 \\
\hline
\end{tabular}

Fuente: Anuarios estadístico y geográfico de Jalisco y de Nayarit 2017 (INEGI, 2019). 
Puerto Vallarta es casi exclusivamente una zona urbana, el $90 \%$ de la población vive en la ciudad, Bahía de Banderas es un mosaico de poblaciones distribuidas en todo su territorio, con zonas rurales tradicionales (Valle de Banderas y San Juan de Abajo), nuevos poblamientos suburbanos, tipo dormitorio entrelazados con poblaciones tradicionales (Mezcales, San Vicente, Jarretaderas), áreas de playa tradicionales con poblaciones emblemáticas (Sayulita, San Francisco y Bucerias), y zonas de turismo exclusivo (Nuevo Vallarta y Punta Mita- Litibu).

El crecimiento poblacional, además de la tasa natural de natalidad, es resultado de una creciente migración de personas que son atraídas por la oferta de trabajo que generan los proyectos turísticos, la construcción de infraestructura y las actividades complementarias al turismo.

La mayor parte de los migrantes provienen de las zonas campesinas pauperizadas o de otras regiones del interior de los estados (tabla 10), La población de la región es de forma mayoritaria mestiza, no se tienen poblaciones originales asentadas en el territorio, salvo grupos migrantes de pueblos originarios del sur de país.

Tabla 10. Población según lugar de residencia (porcentaje para el año de 2015).

\begin{tabular}{|c|c|c|}
\hline & $\begin{array}{c}\text { Puerto } \\
\text { Vallarta }\end{array}$ & $\begin{array}{c}\text { Bahía de } \\
\text { Banderas }\end{array}$ \\
\hline Nacido en el mismo estado & 92.02 & 83.55 \\
\hline Nacidos en otro estado & 7.44 & 16.15 \\
\hline No especificado & 0.54 & 0.30 \\
\hline
\end{tabular}

Fuente: Anuarios estadístico y geográfico de Jalisco y de Nayarit 2017 (INEGI, 2019).

Una condición que diferencia a ambas localidades el número de unidades económicas instaladas y el personal ocupado, en ambos casos es más del doble en Puerto Vallarta, tomando en consideración que muchos trabajadores tienen su residencia en Bahía de Banderas y se desplazan a Puerto Vallarta, donde existe mayor oferta de trabajo (tabla 11).

Tabla 11. Unidades económicas y Población ocupada (Total en 2015).

\begin{tabular}{|l|c|c|}
\hline & $\begin{array}{l}\text { Puerto } \\
\text { Vallarta }\end{array}$ & $\begin{array}{c}\text { Bahía de } \\
\text { Banderas }\end{array}$ \\
\hline Unidades económicas & 12,247 & 5,912 \\
\hline Personal ocupado & 51,872 & 24,560 \\
\hline \% de la población Total & 18.8 & 16.3 \\
\hline
\end{tabular}

Fuente: Anuarios estadístico y geográfico de Jalisco y de Nayarit 2017 (INEGI, 2019).
En cuanto a la distribución de la población por los sectores productivos, es mayor el porcentaje de personas ocupadas en los sectores de comercio y servicio en PVR y en el sector agrícola e industrial en BB (la industria se concentra en la construcción), por su estructura geográfica, es notable que una parte importante de la población se dedica a actividades agrícolas, de forma particular en la zona del Valle (Tabla 12).

Tabla 12. Porcentaje de la población ocupada por sector económico (2015)

\begin{tabular}{|c|c|}
\hline 12Puerto Vallarta & Bahía de Banderas \\
\hline 1.66 & 6.17 \\
\hline 12.28 & 15.04 \\
\hline 19.13 & 16.69 \\
\hline 65.25 & 61.43 \\
\hline 0.93 & 0.51 \\
\hline
\end{tabular}

Fuente: Anuarios estadístico y geográfico de Jalisco y de Nayarit 2017 (INEGI, 2019).

Los servicios públicos (agua, drenaje y luz eléctrica) en ambos municipios tienen una cobertura mayor al $99 \%$, lo que representa un alto nivel de desarrollo humano basado en los servicios (INEGI 2019).

La población de estudio en la región tiene una estructura demográfica y económica relacionada con el turismo, por el número de empresas y empleados que se dedican en este sector, la mayor parte de los establecimientos de comercio y servicio tienen esta orientación, por el número de migrantes atraídos por el trabajo asociado al turismo, también explica una mayor capacidad socioeconómica en Puerto Vallarta, aunque Bahía de Banderas tiene un crecimiento porcentual mayor, lo que permite inferir que las brechas se cerraran.

Esta información permite demostrar la importancia que tiene conocer su percepción sobre los impactos del turismo de los residentes de la región, que sirva como un insumo en los procesos de planificación del turismo y de ciudad.

\section{RESULTADOS}

\subsection{Análisis Factorial}

Para ambas muestras el índice de Kaiser Mayer Olkin se encuentran entre 0.5 y 1.0 y la prueba de esfericidad de Bartlett tiene un valor de significación menor a 0.05, lo que indican que la información es válida y se puede realizar el análisis factorial (Tabla 13). 
Tabla 13. KMO y Test de Bartlet de las muestras

\begin{tabular}{|l|l|c|c|}
\hline \multicolumn{2}{|l|}{ Prueba de KMOy Bartlett } & Puerto Vallarta & Bahía de Banderas \\
\hline Medida Kaiser-Meyer-Olkin de adecuación de muestreo & & .759 & .727 \\
\hline Prueba de esfericidad de Bartlett & Aprox. Chi-cuadrado & 2268.232 & 1767.189 \\
\hline & Gl & 78 & 78 \\
\hline & Sig. & 0.000 & 0.000 \\
\hline
\end{tabular}

Fuente: elaboración propia a partir de los resultados de la encuesta.

Se presenta la matriz de componentes rotados para ambas localidades (Tablas 14 y 15), que determinan cinco componentes (factores), los cuales coinciden con lo determinado teóricamente, el primer componente se le denomina "problemas sociales", integrado por las variables drogadicción, prostitución y alcoholismo, el segundo componente se denomina "problemas culturales" que lo integran las variables de tradición e identidad, el tercer componente se denomina "problemas medioambientales" que lo integran las variables de bosques, ecosistemas y erosión, el cuarto componente se denomina "percepción", que lo integran las variables de interés y participación (este componente, no es un problema, se incluye porque servirá para tener una variable endógena donde medir la participación) y el quinto componte serán los "problemas económicos" que se integra con las variables empleo y empleo remunerado.

En la tabla se presentan los valores de las comunalidades, que es la varianza que una variable comparte con las demás y cuyos valores de todas las variables son superiores a 0.6 que es minimo satisfactorio (de hecho, en el proceso del trabajo se eliminaron variables que no cumplieron este criterio).

Se integran los valores del Alpha de Cronbach, que es un estadístico de fiabilidad de las escalas usadas en el instrumento para cada uno de los factores y de forma total, teniendo valores por arriba de 0.6, situación que es satisfactoria en ambas muestras.

Tabla 14. Matriz de componente rotado, Comunalidades y Estadístico de Fiabilidad de Puerto Vallarta ${ }^{\text {. }}$

\begin{tabular}{|c|c|c|c|c|c|c|c|c|c|}
\hline \multirow[b]{2}{*}{ Factores } & \multirow[b]{2}{*}{ Variables } & \multicolumn{5}{|c|}{ Componente } & \multirow{2}{*}{$\begin{array}{l}\text { Comunalidades } \\
\text { Extracción }\end{array}$} & \multicolumn{2}{|c|}{ Estadísticas de fiabilidad } \\
\hline & & 1 & 2 & 3 & 4 & 5 & & $\begin{array}{l}\text { Alpha de } \\
\text { Cronbach }\end{array}$ & $\begin{array}{c}\mathrm{Nde} \\
\text { elementos }\end{array}$ \\
\hline \multirow{3}{*}{ Sociales } & Drogadicción & .848 & .167 & .184 & -.097 & .080 & .797 & \multirow{3}{*}{.837} & \multirow{3}{*}{ 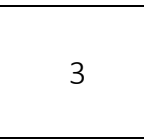 } \\
\hline & Prostitución & .821 & .185 & .051 & .185 & .080 & .751 & & \\
\hline & Alcoholismo & .815 & .130 & .142 & -202 & .121 & .757 & & \\
\hline \multirow{2}{*}{ Culturales } & Tradición & .184 & .878 & .088 & -.079 & .153 & .841 & \multirow{2}{*}{.872} & \multirow{2}{*}{2} \\
\hline & Identidad & .229 & .819 & .130 & -.043 & .183 & .777 & & \\
\hline \multirow{3}{*}{$\begin{array}{l}\text { Medio } \\
\text { ambientales }\end{array}$} & Bosques & .082 & .110 & .874 & -.004 & -.016 & .782 & \multirow{3}{*}{.774} & \multirow{3}{*}{3} \\
\hline & Ecosistemas & .099 & 121 & .800 & .070 & 159 & .694 & & \\
\hline & Erosión & .140 & -.016 & .771 & .081 & .086 & .628 & & \\
\hline \multirow{2}{*}{ Participación } & Interés & -.050 & .035 & -.005 & .866 & .141 & .773 & \multirow{2}{*}{.730} & \multirow{2}{*}{2} \\
\hline & Participas & .037 & .034 & .140 & .852 & .088 & .755 & & \\
\hline \multirow{2}{*}{ Económicos } & Empleo & .082 & .080 & .080 & .168 & .831 & .738 & \multirow{2}{*}{.670} & \multirow[b]{2}{*}{2} \\
\hline & Remunerado & .109 & .212 & .116 & .066 & .768 & .665 & & \\
\hline & Total & & & & & & Total & .776 & 12 \\
\hline
\end{tabular}

Método de extracción: análisis de componentes principales. Método de rotación: Varimax con normalización Kaiser. ${ }^{a}$ a. La rotación ha convergido en 5 iteraciones

Fuente: elaboración propia a partir de los resultados de la encuesta.

Tabla 15. Matriz de componente rotado, Comunalidades y Estadístico de Fiabilidad de Bahía de Banderas.

\begin{tabular}{|c|c|c|c|c|c|c|c|c|c|}
\hline & & Comp & nente & & & & Comunalidades & Estadística & de fiabilidad \\
\hline Factores & Variables & 1 & 2 & 3 & 4 & 5 & Extracción & $\begin{array}{c}\text { Alfa de } \\
\text { Cronbach }\end{array}$ & $\begin{array}{c}\mathrm{N} \text { de } \\
\text { elementos }\end{array}$ \\
\hline \multirow{3}{*}{ Sociales } & drogadicción & .871 & .098 & .094 & .043 & -.006 & .779 & \multirow{3}{*}{.843} & \multirow{3}{*}{3} \\
\hline & Alcoholismo & .839 & .080 & .182 & .047 & .046 & .747 & & \\
\hline & Prostitución & .824 & .060 & -.006 & .006 & .018 & .683 & & \\
\hline
\end{tabular}




\begin{tabular}{|c|c|c|c|c|c|c|c|c|c|}
\hline \multirow{2}{*}{$\begin{array}{l}\text { Medio } \\
\text { Ambientales }\end{array}$} & Bosques & .126 & .838 & .133 & .032 & .017 & .737 & \multirow{3}{*}{.790} & \multirow{3}{*}{3} \\
\hline & Ecosistemas & .115 & .819 & .251 & .033 & -.050 & .751 & & \\
\hline \multirow{3}{*}{ Culturales } & Erosión & .109 & .793 & -.031 & -.052 & .153 & .668 & & \\
\hline & Tradiones & .083 & .138 & .908 & -.022 & -.042 & .843 & \multirow{2}{*}{.821} & \multirow{2}{*}{2} \\
\hline & Identidad & .257 & .151 & .866 & -.007 & .061 & .853 & & \\
\hline \multirow{2}{*}{ Participación } & Interés & .050 & -.054 & -.019 & .871 & .106 & .775 & \multirow{2}{*}{.677} & \multirow{2}{*}{2} \\
\hline & Participas & .093 & .056 & -.005 & .858 & -.008 & .748 & & \\
\hline \multirow{3}{*}{ Económicos } & remunerados & .032 & -.093 & .059 & -.004 & .843 & .724 & \multirow{2}{*}{.613} & \multirow{2}{*}{2} \\
\hline & Empleos & -.044 & .227 & -.054 & .108 & .781 & .678 & & \\
\hline & Total & & & & & & & .716 & 12 \\
\hline
\end{tabular}

Método de extracción: análisis de componentes principales. Método de rotación: Varimax con normalización Kaiser. ${ }^{a}$ a.

La rotación ha convergido en 5 iteraciones.

Fuente: elaboración propia a partir de los resultados de la encuesta.

\subsection{Construcción del modelo estructural}

A partir de la información generada en el análisis factorial, se realiza un Modelo de Ecuaciones Estructurales (SEM) (figura 1), que se pretende pruebe el objetivo del trabajo si la percepción de los impactos del turismo es un preditor de la participación de la población, a partir de cuatro factores latentes (impactos sociales, económicos, culturales y ambientales), estas cuatro variables latentes exógenas son el predictor de una nueva variable latente endógena, la cual se denomina percepción, el cual se relaciona con la el factor latente endógeno denominado participación que es a su vez determinado por los variables observadas interés y participación.

Figura 1. Construcción del modelo de ecuaciones estructurales.

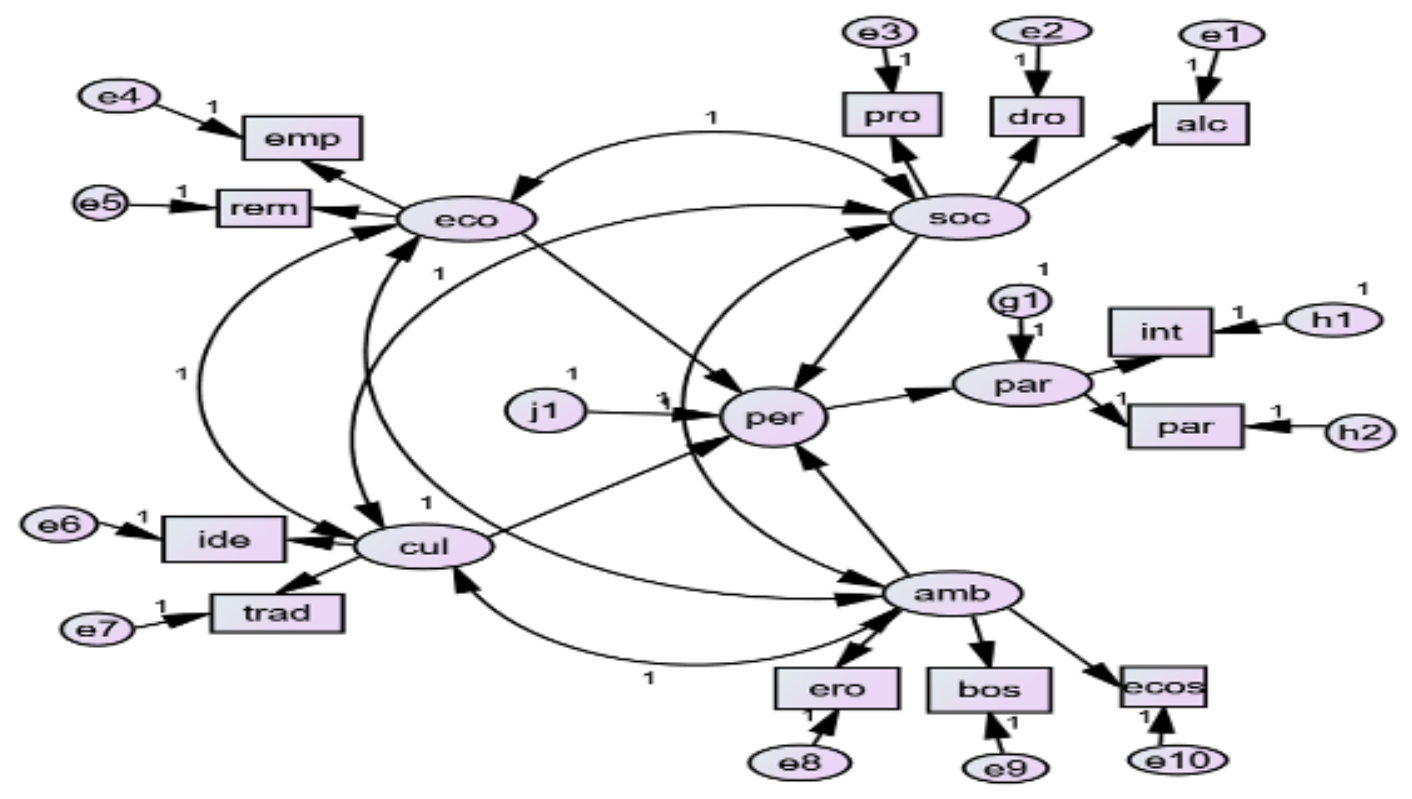

Fuente: elaboración propia de la construcción del modelo.

Una hipótesis adicional es que al comparar los resultados de los modelos se pueda identificar sí existen existen diferencias en ambas localidades a partir del resultado del modelo. En la construcción del modelo se tienen elementos que permiten tomar las cargas no reconocidas por las variables que se usan, se denominan errores de medida que están asociados a las variables observables y errores de predicción que están asociados a las variables latentes endógenas, las variables exógenas no tienen este tipo de errores, pero se explican adicionalmente con la covariación que se observa en el modelo (a través de las flechas bidireccionales entre ellas) (Arburkle, 2015; Hair, Jet al., 2008). Se tiene un resumen de los tipos de variables y parametros que se requirieron en su elaboración que identifican al número de grados de error (tabla 16). 
Tabla 16. Análisis de las Variables y computación de grados de error

\begin{tabular}{|l|c|c|}
\hline & Puerto Vallarta & Bahía de Banderas \\
\hline Number of variables in your model: & 32 & 32 \\
\hline Number of observed variables: & 12 & 12 \\
\hline Number of unobserved variables: & 20 & 20 \\
\hline Number of exogenous variables: & 18 & 18 \\
\hline Number of endogenous variables: & 14 & 14 \\
\hline \multicolumn{2}{|c|}{ Computation of degrees of freedom (Default model) } \\
\hline Number of distinct sample moments: & 78 & 78 \\
\hline Number of distinct parameters to be estimated: & 32 & 32 \\
\hline Degrees of freedom (78-32) & 46 & 46 \\
\hline
\end{tabular}

Fuente: elaboración propia a partir de los resultados del modelo.

De acuerdo a la tabla de los índices de ajuste, todos los valores obtenidos para ambos modelos son satisfactorios, por tanto es idóneo la realización del modelo de ecuaciones estructurales.

Tabla 17. Índices de bondad de ajuste del modelo y valores para considerar su idoneidad

\begin{tabular}{|c|c|c|c|}
\hline \multirow{2}{*}{ Índice de Ajuste } & \multicolumn{2}{|c|}{ Valor obtenido } & \multirow{2}{*}{ Valor idóneo } \\
\cline { 2 - 3 } & Puerto Vallarta & Bahía de Banderas & Mejor cuando más pequeño \\
\hline $\mathrm{x}^{2}$ & 118.790 & 91.188 & Menos a 5 \\
\hline $\mathrm{CMI} / \mathrm{DF}$ & $118.790 / 46=2.582$ & $91.188 / 46=1.982$ & Superior 0.9, mejor cuando más se acerque a 1 \\
\hline $\mathrm{GFI}$ & 0.954 & 0.965 & $\begin{array}{l}\text { Mejor cuando más se acerque a 1, supone un } \\
\text { mejor ajuste con valores arriba de 0.95 }\end{array}$ \\
\hline $\mathrm{CFI}$ & 0.958 & 0.970 & Un valor entre 0.05 y 0.08 indica un buen ajuste \\
\hline REMSEA & 0.063 & 0.050 &
\end{tabular}

Fuente: elaboración propia a partir de los resultados del modelo.

El análisis del ajuste global del modelo que reproduce adecuadamente las relaciones en la matriz de covarianzas de los datos empíricos, asegura que es factible el modelo, pues con el número de grados de libertad y los valores de Chi cuadrada que se presentan tienen un estadístico de probabilidad asociado menor a 0.05 que es un valor significativo, esto a pesar que el valor de Chi cuadrada esta sobre estimado debido al tamaño de muestra, por lo que se toman como válidos el resto de los valores de ídoneidad presentados (Tabla 18).

\section{Tabla 18. Resultados de la aplicación del modelo.}

\begin{tabular}{|l|l|}
\hline Puerto Vallarta & Bahía de Bandera \\
\hline Minimum was achieved & Minimum was achieved \\
Chi-square $=118.790$ & Chi-square $=91.188$ \\
Degrees of freedom $=46$ & Degrees of freedom $=46$ \\
Probability level $=.000$ & Probability level $=.000$ \\
\hline
\end{tabular}

Fuente: elaboración propia a partir de los resultados del modelo.

Figura 2. Solución del modelo de Puerto Vallarta

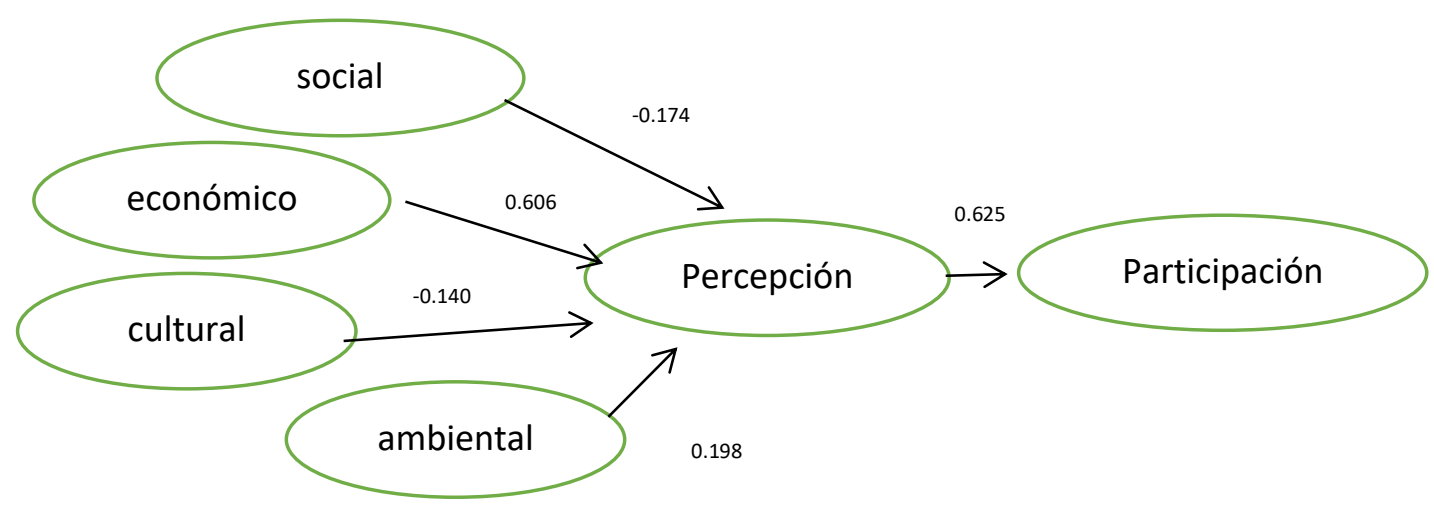

Fuente: elaboración propia a partir de los resultados del modelo. 
Figura 3. Solución del modelo de Bahía de Banderas

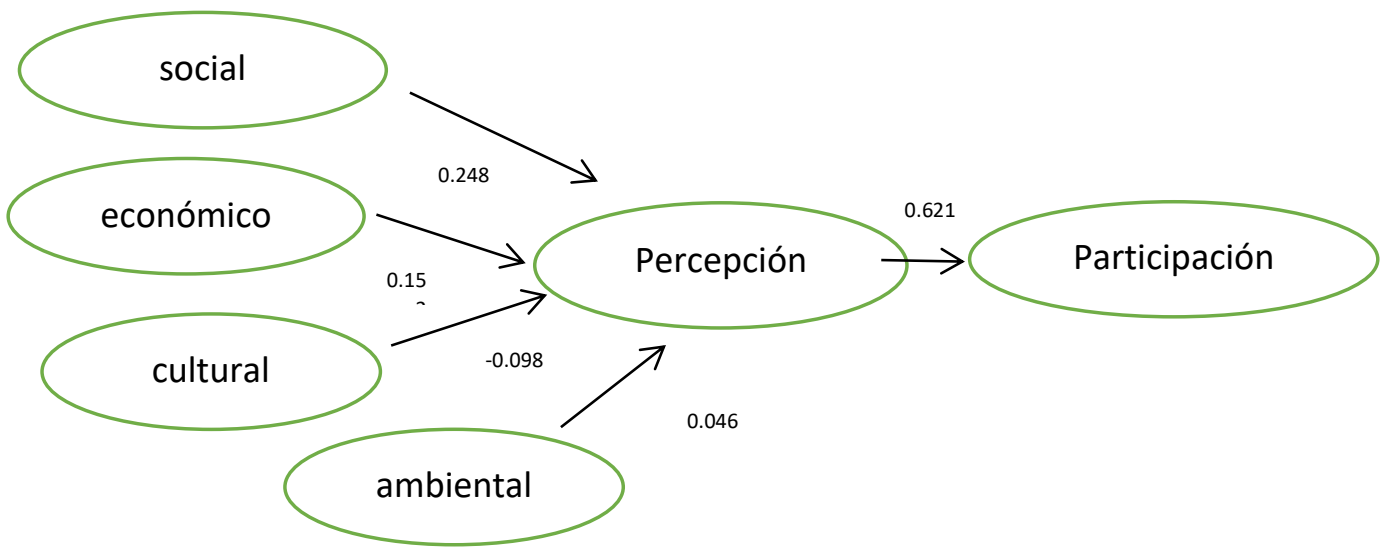

Fuente: elaboración propia a partir de los resultados del modelo.

Los resultados de las pruebas estadísticas que se presentan permiten que la costrucción de los modelos para cada localidad sean adecuados (figura 2 y3), en las cuales se presentan una versión abreviada que permita ser analizada de forma simple y (tablas 19 y 20) donde se integran todos los valores del modelo para un mejor análisis.

Tabla 19. Valores obtenidos de la aplicación del modelo de Puerto Vallarta

\begin{tabular}{|c|c|c|c|c|c|c|c|}
\hline & & & Estimate & S.E. & C.R. & P & Estimate \\
\hline percepcion & $<-$ & Sociales & -.199 & .147 & -1.352 & .176 & -.174 \\
\hline percepcion & $<-$ & Economicos & 1.058 & .480 & 2.204 & .028 & .606 \\
\hline percepcion & $<-$ & Culturales & -.148 & .146 & -1.011 & .312 & -.140 \\
\hline percepcion & $<-$ & ambientales & .481 & 292 & 1.650 & . 099 & .198 \\
\hline participacion & $<-$ & percepcion & .630 & 193 & 3.265 & .001 & .625 \\
\hline drogas & $<-$ & Sociales & 1.185 & .066 & 18.059 & $* * *$ & .927 \\
\hline Alcohol & $<-$ & Sociales & 1.000 & & & & .815 \\
\hline remunerado & $<-$ & economicos & 1.000 & & & & .590 \\
\hline empleos & $<-$ & economicos & 1.000 & & & & .674 \\
\hline tradición & $<-$ & culturales & 1.000 & & & & .852 \\
\hline identidad & $<-$ & culturales & 1.030 & .076 & 13.537 & $* * *$ & .908 \\
\hline erosión & $<-$ & ambientales & 1.000 & & & & .639 \\
\hline ecosistemas & $<-$ & ambientales & 1.473 & 136 & 10.863 & $* * *$ & .759 \\
\hline interés & $<-$ & participacion & .625 & .077 & 8.158 & $* * *$ & .625 \\
\hline participas & $<-$ & participacion & 1.000 & & & & .903 \\
\hline bosques & $<-$ & ambientales & 1.347 & .117 & 11.473 & $* * *$ & .814 \\
\hline prostitución & $<-$ & Sociales & .902 & .065 & 13.959 & $* * *$ & .666 \\
\hline
\end{tabular}

Fuente: elaboración propia a partir de los resultados del modelo.

Tabla 20. Valores obtenidos de la aplicación del modelo de Bahía de Banderas

\begin{tabular}{|c|c|c|c|c|c|c|c|}
\hline & & & Estimate & S.E. & C.R. & P & Estimate \\
\hline percepcion & & Sociales & .227 & .117 & 1.935 & .053 & .248 \\
\hline percepcion & $<-$ & Economicos & .236 & .223 & 1.060 & .289 & 152 \\
\hline percepcion & $<-$ & Culturales & -.115 & .120 & -.960 & .337 & -.098 \\
\hline percepcion & $<-$ & ambientales & .072 & .167 & .432 & .666 & .046 \\
\hline participacion & $<-$ & percepcion & .758 & .218 & 3.483 & $* * *$ & .621 \\
\hline Drogas & $<-$ & Sociales & 1.093 & .061 & 18.041 & $* * *$ & .895 \\
\hline Alcohol & & Sociales & 1.000 & & & & .862 \\
\hline remunerado & & economicos & 1.000 & & & & .558 \\
\hline
\end{tabular}




\begin{tabular}{|c|c|c|c|c|c|c|}
\hline & & Estimate & S.E. & C.R. & P & Estimate \\
\hline Empleos & $<--\quad$ economicos & 1.000 & & & & .627 \\
\hline Tradición & $<-\quad$ culturales & 1.000 & & & & .704 \\
\hline Identidad & $<-\quad$ culturales & 1.415 & .176 & 8.041 & $* * *$ & .989 \\
\hline Erosión & $<-$ ambientales & 1.000 & & & & .619 \\
\hline ecosistemas & $<-$ ambientales & 1.527 & .137 & 11.122 & $* * *$ & .818 \\
\hline Interés & $<-\quad$ participacion & .496 & .096 & 5.179 & $* * *$ & .535 \\
\hline Participas & $<-$ participacion & 1.000 & & & & .953 \\
\hline Bosques & $<-\quad$ ambientales & 1.496 & .130 & 11.495 & $* * *$ & .805 \\
\hline prostitución & $<-\quad$ Sociales & .803 & .058 & 13.829 & $* * *$ & .658 \\
\hline
\end{tabular}

Fuente: elaboración propia a partir de los resultados del modelo.

A partir de esta información se puede contestar la hipótesis general, que determina que la percepción de los problemas, determinados por cuatro variables latentes (problemas sociales, económicos, culturales y ambientales) es un predictor de la participación, medido por dos variables latentes (interés por participar y haber participado en la solución de problemas), teniendo valores estandarizados en Puerto Vallarta (0.625) y en Bahía de Banderas (0.621).

En cuanto a la construcción de las variables latentes sobre los impactos se tiene para Puerto Vallarta que los impactos sociales tienen un valor estandarizado de -0.174 , los impactos económicos tienen un valor estandarizado de 0.606 , la percepción cultural de - 0.140 y los impactos ambientales de 0.198 , a pesar de que las cargas son bajas si permite explicar que tienen influencia sobre la percepción de la población local.

Para Bahía de Banderas los impactos sociales tienen un valor estandarizado de 0.248 , los impactos económicos tienen un valor estandarizado de 0.152 , la percepción cultural de -0.098 y los impactos ambientales de 0.046, a pesar de que las cargas son bajas si permite explicar que tienen influencia sobre la percepción de la población local.

De manera adicional se prueba que en Puerto Vallarta este predictor es mayor que el que se estima para Bahía de Banderas (aunque la diferencia en apenas de 0.04) que no es signigficativa, por lo que se puede concluir que no existen diferencias en los dos municipios.

En cuanto a la construcción de las variables latentes (exógenas) que se integran a partir de las variables independientes, se pueden explicar como respuesta a hipótesis adicionales, se puede inferir que en el factor sociedad la drogadicción y la prostitución tiene una mayor percepción en Puerto Vallarta y el alcoholismo es mayormente percibido en Bahía de Banderas.
En el factor de economía tiene más peso la variable de falta de empleos en Puerto Vallarta y en Bahía de Banderas tiene mayor peso la falta de empleos bien remunerados.

En la variable de cultura tiene mayor peso la pérdida de tradiciones en Puerto Vallarta y en Bahía de Banderas la pérdida de identidad.

Para el factor de medio ambiente, la variable de pérdida de ecosistemas marítimos y terrestres tiene más peso en Bahía de Banderas, mientras que las variables de erosión y pérdida de bosques y playas tienen más peso en Puerto Vallarta.

Este trabajo no tiene la pretensión de determinar el grado de percepción negativa del turismo, tomando al turismo como una actividad que involucra tanto a los turistas, habitantes locales, prestadores de servicios turísticos (tanto propietarios como trabajadores) y a los diversos niveles de gobierno, solo toma en consideración a los residentes del destino.

\subsection{Discusión}

El tema que se presenta tiene la fortuna de ser estudiado desde muy variadas disciplinas científicas y desde diversos abordajes metodológicos, la aportación de este trabajo es que se realizó en un destino turístico consolidado Puerto Vallarta- Bahía de Banderas, que tiene más de cincuenta años con un crecimiento turístico casi sostenido, medido por el número de visitantes y un crecimiento poblacional asociado al desarrollo del turismo.

El principal hallazgo que se desprende de la investigación es que la población local es un actor indispensable en el desarrollo del turismo, para integrarse en los procesos de mejora se debe reconocer la existencia de problemas y a partir de ello provocar los mecanismos de solución estudios 
anteriores han confirmado lo anterior como los que proponen Nunkoo y Gursoy (2012), Rasoolimanesh, Jaafar, et al. (2017), Ribeiro et al. (2017), VargasSánchez et al. (2011) y Eusébio et al. (2018).

El modelo genera que la pecepción asociada al turismo por parte de los residentes es un predictor del interés por particpar en la solición de los problemas, y se le asocia un valor estandarizado de 0.625 en el estudio de Puerto Vallarta y de 0.621 en el estudio de Baía de Banderas, con los que se prueba para ambas pruebas la hipótesis propuesta.

Se valida lo determinado por la Teoría del intercambio social donde se establece que los actores del proceso social, en este caso el turismo, están de acuerdo en intercambiar sus aportaciones individuales y colectivas, a pesar de los costos que ello implica que es recompensado por los beneficios obtenidos, en favor de trabajar en beneficio del desarrollo turístico sostenible como lo postulan Ap (1992), Gursoy y Rutherford (2004), Ko y Stewart (2002), Nunkoo y Gursoy (2012), Vargas-Sánchez et al. (2011) y Andereck y Vogt (2000), este trabajo abona en ese sentido, la sociedad de la región PVR-BB, conoce de los impactos del turismo y está dispuesta a trabajar en la busqueda de soluciones, entendiendo en cada localidad los beneficios y los costos son percibidos diferentes y así como el nivel de desarrollo de la actividad turística.

Las diferencias de la percepción entre ambos municipios conurbados no son significativas, siendo en general más alta la preocupación de los habitantes de Puerto Vallarta, pero éstas diferencias no cambian el sentido de la conclusión para ninguno de las localidades, solo permite entender sus condiciones particulares, de acuerdo con Rasoolimanesh, Ringle, et al (2017) y Vargas-Sánchez et al. (2011). Aunque en el trabajo que se presenta algunas variables se comportan de manera diferente en ambas localidades.

Una condición que es fundamental es que la relación de los habitantes locales y los turistas sucede en un espacio, que es parte de la comunidad o que ha sido construido para la realización del turismo y los locales lo compartan y perciben los efectos positivos y negativos de ello, como son la mejora en su infraestructura, la degradación medio ambiental o la pérdida de espacios de recreo (playas y bosques), como lo refieren Campodónico (2016) e lqbal (2020).

La población identifica que los factores económicos, sociales, culturales y medioambientales son parte de la percepción que tiene sobre la actividad turística, en ella identifican los impactos positivos y negativos y reflejan lo que colectivamente construyen como comunidad, en concordancia con los resultados de los trabajos de Nunkoo y Gursoy (2012), Rasoolimanesh, Jaafar, et al. (2017), Ribeiro el al. (2017) Vargas-Sánchez et al. (2011) y Eusebio et al. (2018).

La importancia que la sociedad le da a los factores económicos sobre los otros tres que se han estudiado (sociales, culturales y económicos), tienen una correspondencia con los resultados de las investigaciones de Ap (1992), Gursoy y Rutherford (2004), Ribeiro el al. (2017) Yu et al. (2018) Eusebio et al. (2018).

Una cuestión que está en el centro de la discusión es sí la construcción de la muestra estadística en un lugar y tiempo determinado, bajo los criterios de selección, es suficiente para establecer que representa a la percepción de toda la población, situación que debe ser tomada con precaución, aunque los resultados son coincidentes con otros estudios similares, se debe apuntar que los resultados se basan en estimaciones estadísticas que bajo las condiciones temporales y espaciales determinadas arrojaron los resultados descritos, pero que en otro momento o lugar estos pueden ser diferentes, porque las condiciones sociales cambiaron o los grupos sociales que se analizan tienen diferentes construcciones sociodemográficas y por tanto son diferentes sus percepciones, como lo explicó Sharpley (2014).

Un aporte metodológico es que este modelo que ya a sido usado en trabajos previo (Gauna, 2017), se ha mejorado en su construcción y permite tener una mejor comprensión de la realidad, haciendo un mejor aporte cuando se trabaja en dos poblaciones y se logran criterios de idoneidad de ajuste y por tanto se refuerza su validez como instrumento.

La compresión de la realidad de un territorio se va construyendo con el trabajo que desde diversos abordajes se realiza, cuando estos trabajos presentan coincidencias se tienen mejores elementos para poder resolver los problemas que se han planteado en los estudios empíricos. En el tiempo si no se mejoran las condiciones de la sociedad o incluso si estas continúan generando afectaciones graves, la percepción de la población será aún más crítica, por lo que los tomadores de decisión deben trabajar en los cambios favorables que se requieren para mantener el liderazgo que el destino turístico ha tenido hasta ahora. 


\section{CONCLUSIONES}

El trabajo que se presenta contesta la pregunta básica, la población participa en la solución de sus problemas a partir del reconocimiento de la existencia de impactos que afectan su entorno, esto es más valido cuando la actividad que se realiza en el territorio es el turismo.

La percepción de la población sobre los impactos que genera el turismo es un predictor del interés por participar en la búsqueda de soluciones de los problemas de la comunidad, esta es la hipótesis del trabajo, la cual se responde de manera afirmativa, lo que implica qué, a mayor conocimiento de la problemática asociada al turismo, las personas tienen un mayor interés por la participación social.

Esto se demuestra de igual forma en las dos comunidades estudiadas, las diferencias estadísticas entre ambas muestras no son significativas, por lo que se concluye que en ambas comunidades existe la preocupación por los problemas y que el interés por participar es determinado por este conocimiento Nunkoo y Gursoy (2012), Rasoolimanesh, Jaafar et al. (2017), Ribeiro el al. (2017) Vargas-Sánchez et al. (2011) Eusebio et al. (2018).

El destino Puerto Vallarta ha crecido de manera sostenida y esto ha llevado a una ampliación del espacio turístico que ahora abarca el territorio de dos municipios Puerto Vallarta, Jalisco y Bahía de Banderas, Nayarit, ha caracterizado a cada una de las poblaciones de forma particular, a pesar de formar parte de la misma zona metropolitana y tener problemas comunes, se tienen comunidades locales con algunas diferencias que los identifiquen.

El trabajo que se presenta abona a la comprensión de las preocupaciones e intereses de la población, existen muchas cuestiones que deben ser analizadas desde otros estudios, que podrán ser abordados desde diversas metodologías que permitan tener una comprensión mejor de la realidad, el trabajo además se suma a otros trabajos que explican desde otras posturas el papel que juegan las poblaciones locales en el proceso de la actividad turística.

Para posteriores trabajos este modelo empírico se puede aplicar en otros territorios similares, incluso se puede mejorar al incluir otras variables que mejoren el modelo que ahora se presenta, o que se genere una orientación particular, a partir de la nueva construcción que este fundamentada en otras aportaciones empíricas que trabajan sobre similares problemáticas.
También se deben generar investigaciones particulares por grupos de la población, género, edad, tiempo de residencia y nivel de participación productiva en el turismo.

Otra consideración para posteriores trabajos tiene que ver con analizar las formas como la sociedad participa o debe participar en la solución de los problemas, en los procesos de planeación del desarrollo y en la implementación de políticas públicas que abonen a mejorar su calidad de vida y el bienestar de toda la población.

\section{REFERENCIAS}

Andereck, K. L., Valentine, K. M., Knopf, R. C., \& Vogt, C. A. (2005). Residents' perceptions of community tourism impacts. Annals of Tourism Research, 32(4), 10561076. https://doi.org/10.1016/j.annals.2005.03.001

Andereck, K. L., \& Vogt, C. A. (2000). The relationship between residents' attitudes toward tourism and tourism development options. Journal of Travel Research. https://doi.org/10.1177/004728750003900104

Andriotis, K., \& Vaughan, R. D. (2003). Urban residents' attitudes toward tourism development: The case of Crete. Journal of Travel Research, 42(2), 172-185. https://doi.org/10.1177/0047287503257488

Ap, J. (1992). Residents' perceptions on tourism impacts. Annals of Tourism Research. https://doi.org/10.1016/0160-7383(92)90060-3

Arburkle, J. (2015). Amos 23.0 User Guide. (A. D. Corporation, Ed.). E. U. A.

Campodónico, R. (2016). Turismo: De La Movilidad Al Espacio. Revista Latino-Americana de Turismologia, 1(2), 8-16. Recuperado http://ojs2.ufff.emnuvens.com.br/rlaturismologia/artic le/view/9999

Doxey, G. V. (1975). A causation theory of visitor-resident irritants: Methodology and research inferences. Proceedings... Sixth Annual TTRA Conference.

Eusébio, C., Vieira, A. L., \& Lima, S. (2018). Place attachment, host-tourist interactions, and residents' attitudes towards tourism development: the case of Boa Vista Island in Cape Verde. Journal of Sustainable Tourism, 26(6), 890-909. https://doi.org/10.1080/09669582.2018.1425695

Gauna, C. (2017). Percepción de la problemática asociada al turismo y el interés por participar de la población: caso Puerto Vallarta. Revsita Periplo Sustentable No. 33, 251290.

Gursoy, D., \& Rutherford, D. G. (2004). Host attitudes toward tourism: An improved structural model. Annals of Tourism Research, 31(3), 495-516. https://doi.org/10.1016/j.annals.2003.08.008

Hair, J., Anderson, R., Tathan, R., \& Black, W. (2008). Análisis multivariante. Pearson, Prenstice Hall. 
Harrill, R. (2004). Residents' attitudes toward tourism development: A literature review with implications for tourism planning. Journal of Planning Literature. https://doi.org/10.1177/0885412203260306

INEGI. (2010). INEGl. Recuperado de https://www.inegi.org.mx/

Iqbal, Z. (2020). The application of SEM technique for the assessment of factors influencing Local community members in destination development: A study of the district of Jammu and Kashmir - India. Revista Anais Brasileiros de Estudos Turísticos, 10, 1-12.

Juric, J., Lindenmeier, J., \& Arnold, C. (2020). Do Emotional Solidarity Factors Mediate the Effect of Personality Traits on the Inclination to Use Nonmonetary Peer-toPeer Accommodation Networks? Journal of Travel Research. https://doi.org/10.1177/0047287519895127

Ko, D. W., \& Stewart, W. P. (2002). A structural equation model of residents' attitudes for tourism development. Tourism Management. https://doi.org/10.1016/S0261-5177(02)00006-7

Kuščer, K., \& Mihalič, T. (2019). Residents' Attitudes towards Overtourism from the Perspective of Tourism Impacts and Cooperation-The Case of Ljubljana. Sustainability, 11(6), 1823. https://doi.org/10.3390/su11061823

Liao, C. C., Lin, Y. X., \& Hsieh, H. H. (2019). Satisfaction of indigenous tourism from residents' perspective: A case study in Nantou County, Taiwan. Sustainability (Switzerland), 11(2), 1-12. https://doi.org/10.3390/su11010276

Luque, T. (2000). Técnicas de análisis de datos en investigación de mercados. (Piramide, Ed.). Madrid, España.

Malhortra, N. (2000). Investigación de mercados. (P. H. Person, Ed.). México.

Meimand, S. E., Khalifah, Z., Zavadskas, E. K., Mardani, A., Najafipour, A. A., \& Ahmad, U. N. U. (2017). Residents' attitude toward tourism development: A sociocultural perspective. Sustainability (Switzerland), 9(7). https://doi.org/10.3390/su9071170

Nunkoo, R., \& Gursoy, D. (2012). Residents' support for tourism. An Identity Perspective. Annals of Tourism Research. https://doi.org/10.1016/j.annals.2011.05.006

Nunkoo, R., Smith, S. L. J., \& Ramkissoon, H. (2013). Residents' attitudes to tourism: A longitudinal study of 140 articles from 1984 to 2010. Journal of Sustainable Tourism, 21(1), 5-25. https://doi.org/10.1080/09669582.2012.673621

Olya, H. G. T., Alipour, H., \& Gavilyan, Y. (2018). Different voices from community groups to support sustainable tourism development at Iranian World Heritage Sites: evidence from Bisotun. Journal of Sustainable Tourism, 26(10),

1728-1748 https://doi.org/10.1080/09669582.2018.1511718

Ouyang, Z., Gursoy, D., \& Chen, K. C. (2019). It's all about life: Exploring the role of residents' quality of life perceptions on attitudes toward a recurring hallmark event over time. Tourism Management, 75(April), 99111. https://doi.org/10.1016/j.tourman.2019.04.032

Pizam, A. (1978). Tourism's Impacts: The Social Costs to the Destination Community as Perceived by Its Residents. Journal of Travel Research https://doi.org/10.1177/004728757801600402

Rasoolimanesh, S. M., Jaafar, M., Kock, N., \& Ahmad, A. G. (2017). The effects of community factors on residents' perceptions toward World Heritage Site inscription and sustainable tourism development. Journal of Sustainable Tourism, 25(2), 198-216. https://doi.org/10.1080/09669582.2016.1195836

Rasoolimanesh, S. M., Ringle, C. M., Jaafar, M., \& Ramayah, T. (2017). Urban vs. rural destinations: Residents' perceptions, community participation and support for tourism development. Tourism Management, 60, 147-158. https://doi.org/10.1016/j.tourman.2016.11.019

Rasoolimanesh, S. M., Taheri, B., Gannon, M., Vafaei-Zadeh, A., \& Hanifah, H. (2019). Does living in the vicinity of heritage tourism sites influence residents' perceptions and attitudes? Journal of Sustainable Tourism, 27(9), 1295-1317.

https://doi.org/10.1080/09669582.2019.1618863

Ribeiro, M. A., Pinto, P., Silva, J. A., \& Woosnam, K. M. (2017). Residents' attitudes and the adoption of pro-tourism behaviours: The case of developing island countries. Tourism Management, 61, 523-537. https://doi.org/10.1016/j.tourman.2017.03.004

Sánchez-Fernández, M. D., Álvarez-Bassi, D., \& Cardona, J. R. (2016). Grupos de residentes de punta del este (Uruguay) según su actitud hacia el turismo. Revista Latinoamericana de Turismologia, 2(1), 55-70.

Sharpley, R. (2014). Host perceptions of tourism: A review of the research. Tourism Management. https://doi.org/10.1016/j.tourman.2013.10.007

Vargas-Sánchez, A., Oom do Valle, P., da Costa Mendes, J., \& Silva, J. A. (2015). Residents' attitude and level of destination development: An international comparison. Tourism Management, 48, 199-210. https://doi.org/10.1016/j.tourman.2014.11.005

Vargas-Sánchez, A., Porras-Bueno, N., \& Plaza-Mejía, M. de los Á. (2011). Explaining residents' attitudes to tourism: Is a universal model possible? Annals of Tourism Research. https://doi.org/10.1016/j.annals.2010.10.004

Virgen, C., \& Gauna, C. (2011). Información demográfica y económica de la región de Puerto Vallarta - Bahía de Banderas: datos para la toma de decisiones. TURyDES Turismo y desarrollo local, 4, 1-25.

Wang, S., \& Chen, J. S. (2015). The influence of place identity on perceived tourism impacts. Annals of Tourism Research, 52, 16-28. https://doi.org/10.1016/j.annals.2015.02.016

Woosnam, K. M. (2012). Using emotional solidarity to explain residents' attitudes about tourism and tourism 
development. Journal of Travel Research. communities: Perceived tourism impacts and https://doi.org/10.1177/0047287511410351 community quality of life perspective. Sustainability

Yu, C. P., Cole, S. T., \& Chancellor, C. (2018). Resident support $\quad$ (Switzerland), $10(3)$. for tourism development in rural midwestern (USA) https://doi.org/10.3390/su10030802.

Processo Editorial / Editorial Process / Proceso Editorial

Editor Chefe/ Editor-in-chief / Editor Jefe: PhD Thiago D. Pimentel (UFJF).

Recebido / Received / Recibido: 19.01.2021; Revisado / Revised/Revisado: 16.03.2021-25.04.2021; Aprovado / Approved /

Aprobado: 08.07.2021; Publicado / Published / Publicado (online): 16.08.2021.

Seção revisada às cegas por pares / Double blind review section / Sessión revisada por pares ciegos. 